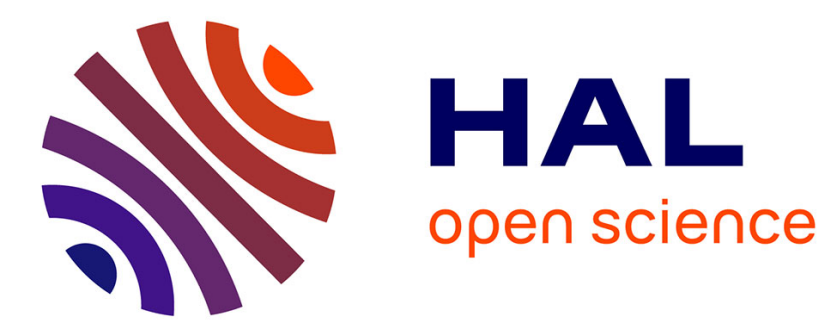

\title{
Effect of the overall rotation on the cis-trans isomerization of HONO induced by an external field
}

\author{
Matthieu Sala, Fabien Gatti, David Lauvergnat, Hans-Dieter Meyer
}

\section{To cite this version:}

Matthieu Sala, Fabien Gatti, David Lauvergnat, Hans-Dieter Meyer. Effect of the overall rotation on the cis-trans isomerization of HONO induced by an external field. Physical Chemistry Chemical Physics, 2012, 14, pp.3791. 10.1039/c2cp23709a . hal-00672953

\section{HAL Id: hal-00672953 https://hal.science/hal-00672953}

Submitted on 14 Jun 2021

HAL is a multi-disciplinary open access archive for the deposit and dissemination of scientific research documents, whether they are published or not. The documents may come from teaching and research institutions in France or abroad, or from public or private research centers.
L'archive ouverte pluridisciplinaire HAL, est destinée au dépôt et à la diffusion de documents scientifiques de niveau recherche, publiés ou non, émanant des établissements d'enseignement et de recherche français ou étrangers, des laboratoires publics ou privés.

\section{(c)(1)}

Distributed under a Creative Commons Attribution| 4.0 International License 


\title{
Effect of the overall rotation on the cis-trans isomerization of HONO induced by an external field
}

\author{
Matthieu Sala, ${ }^{a}$ Fabien Gatti, ${ }^{a}{ }^{a}$ David Lauvergnat ${ }^{b}$ and Hans-Dieter Meyer ${ }^{c}$
}

Rovibrational eigenenergies of HONO are computed and compared to experimental energies available in the literature. For their computation, we use a previously developed potential energy surface (PES) and a newly derived exact kinetic energy operator (KEO) including the overall rotation for a tetra-atomic molecule in non-orthogonal coordinates. In addition, we use the Heidelberg Multi-Configuration Time-Dependent Hartree (MCTDH) package. We compare the experimental rovibrational eigenvalues of HONO available in the literature with those obtained with MCTDH and a previously developed potential energy surface (PES) [F. Richter et al., J. Chem. Phys., 2004, 120, 1306.] for the cis geometry. The effect of the overall rotation on the process studied in our previous work on HONO [F. Richter et al., J. Chem. Phys., 2007, 127, 164315.] leading to the cis $\rightarrow$ trans isomerization of HONO is investigated. This effect on this process is found to be weak.

\section{Introduction}

The typical time scale of the nuclear motion involved in chemical phenomena is of the order of tens to hundreds of femtoseconds. Using so-called "ultrafast" laser pulses that are similar in duration to these processes allows experimentalists to follow dynamical processes in molecules as they happen. ${ }^{1-3}$ This technique opens new possibilities to induce selective chemistry with laser pulses and for the understanding of fundamental phenomena involving the conversion of light into mechanical motion such as the elementary steps of vision, photosynthesis, protein dynamics, and electron and proton transport in DNA. ${ }^{4}$ However, as stated by A. Zewail during his Nobel lecture, the control of chemical reactions by laser pulses could "not be made without knowing and controlling the time scales of intramolecular vibrational energy redistribution (IVR) in molecules" (page 56 in ref. 4). Consequently, general strategies must be developed to provide a complete theoretical description of the energy redistribution that happens before chemical transformations. $^{5-7}$ Unfortunately, describing the kinetics of primary reaction steps in polyatomic molecules by investigation of the underlying full-dimensional quantum dynamics is still a formidable challenge. This is due to the

${ }^{a}$ CTMM, Institut Charles Gerhardt (UMR-CNRS 5253), CC 1501, Université Montpellier II, F-34095 Montpellier, Cedex 05, France.

E-mail:matthieusala@hotmail.fr,gatti@univ-montp2.fr

${ }^{b}$ Laboratoire de Chimie Physique (UMR 8000), Université Paris-Sud, F-91405 Orsay, France.

E-mail: david.lauvergnat@lcp.u-psud.fr

${ }^{c}$ Theoretische Chemie, Universität Heidelberg, Im Neuenheimer Feld 229, D-69120 Heidelberg, Germany.

E-mail: hans-dieter.meyer@pci.uni-heidelberg.de complex structure of vibrational eigenstates, which can be quite dense, and to the presence of vibronic couplings when excited electronic states are to be considered. Advanced quantum dynamics techniques allow us to treat molecules in full dimensionality with up to a maximum of seven atoms typically.

In previous papers, it was demonstrated that the Heidelberg package $^{8}$ of the Multi-Configuration Time Dependent Hartree (MCTDH) algorithm ${ }^{9-12}$ is an efficient tool to investigate IVR in relatively large systems such as toluene ${ }^{13}$ (reduced to 9 degrees of freedom) and fluoroform (9 degrees of freedom), ${ }^{14}$ HFCO,${ }^{15}$ DFCO,${ }^{16}$ and $\mathrm{H}_{2} \mathrm{CS}{ }^{17}$ Besides these systems, we have studied the cis $\rightarrow$ trans isomerization of nitrous acid (HONO) in full dimensionality. ${ }^{18-20}$

In this work, we focus again on the cis $\rightarrow$ trans isomerization of nitrous acid (HONO). Apart from being an important source of $\mathrm{OH}$ radicals in atmospheric chemistry, $\mathrm{HONO}$ is one of the smallest molecules, which shows a cis-trans conformational equilibrium. Its corresponding isomerization exhibits a strong mode selectivity and this molecule constitutes an ideal prototype for theoreticians to investigate selective IVR leading to a chemical process. Consequently, since HONO is a molecule of high environmental and fundamental interest, it has spurred numerous experimental and theoretical works. ${ }^{21-38}$

In a previous paper on $\mathrm{HONO}^{20}$ we have triggered the cis $\rightarrow$ trans isomerization via a laser field. We have simulated the dynamics with MCTDH in the presence of a laser pulse with a carrier frequency of $850 \mathrm{~cm}^{-1}$, a field strength of 0.0035 a.u. $\left(4.3 \times 10^{11} \mathrm{Watt}^{\mathrm{cm}^{-2}}\right)$, and an irradiation duration of $500 \mathrm{fs}$. We have predicted that HONO isomerizes in the gas phase with a yield of about 10 percent when the suggested 
parameters for the pulse are used. This result is remarkable given the fact that it is not the reaction coordinate that is excited by the laser pulse but mainly the central ON stretching mode of vibration. As discussed in ref. 20, an excitation with a carrier frequency near $850 \mathrm{~cm}^{-1}$ seems to be very efficient to promote isomerization. On the other hand, exciting the $\mathrm{O}-\mathrm{H}$ stretching mode leads to almost no isomerization because in a free molecule the $\mathrm{O}-\mathrm{H}$ stretching vibration is almost uncoupled from the other modes. There are several experiments that show isomerization after excitation of the $\mathrm{O}-\mathrm{H}$ stretch. However, these experiments were performed with $\mathrm{HONO}$ in noble gas matrices, where cage effects play a major role.

In ref. 20, the overall rotation of the molecule was neglected. In the present paper, we allow the molecules to rotate. In other words, the process is studied for a given $J \neq 0, J$ being the total angular momentum. We still do not take into account the rotational transitions by absorption of light, i.e. $J$ remains a good quantum number and we do not allow transitions from $J$ to $J^{\prime}$, with $J^{\prime} \neq J$. We will investigate these rotational transitions in future works but in the present paper we focus on another important aspect of dynamics: we now include the fact that the molecules can rotate and that the mode specificity for the process studied in ref. 20 can be influenced by rotation. In particular, whether rotational degrees of freedom couple sufficiently to the vibrational degrees of freedom to mediate the isomerization of HONO in the process studied in ref. 20 or not remains to be investigated, which is the purpose of the present study.

The outline of this paper is as follows. Section II presents the theoretical background. Sections III and IV are devoted to the calculation of the rovibrational eigenstates and to the comparison of the eigenvalues with the experimental data. The following section deals with the effect of the overall rotation on the dynamics. The paper concludes presenting perspectives for the future.

\section{Theory}

\section{A. Coordinates and kinetic energy operator}

As in ref. 19, we represent the geometry of HONO with three valence vectors $\vec{R}_{1}=\overrightarrow{\mathrm{OH}}, \vec{R}_{2}=\overrightarrow{\mathrm{ON}}$ (double bond), and $\vec{R}_{3}=$ $\overrightarrow{\mathrm{ON}}$ (single bond). The three vectors are in turn parametrized by six "polyspherical" coordinates: three distances, $R_{1}, R_{2}, R_{3}$, two planar angles $\theta_{1}, \theta_{2}$, and one dihedral angle $\tau$ as depicted in Fig. 1. As in ref. 19, we use $u_{1}=\cos \theta_{1}$ and $u_{2}=\cos \theta_{2}$ instead of $\theta_{1}$ and $\theta_{2}$. The orientation of the Body-Fixed (BF) frame with

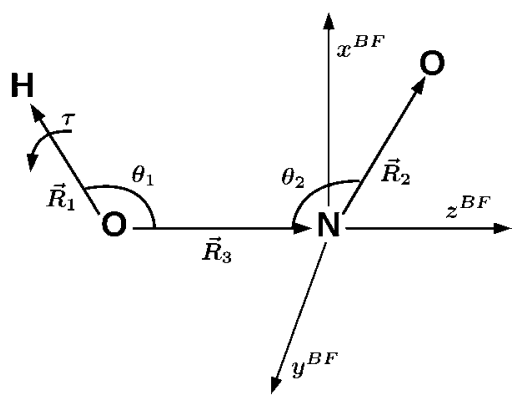

Fig. 1 Valence polyspherical coordinates for the HONO molecule. respect to the Space-fixed (SF) frame is determined by three Euler angles: $\alpha, \beta, \gamma$. The Euler angles are chosen such that the $\mathrm{BF}$ frame is oriented in a way that $\vec{e}_{z} \mathrm{BF}$ is parallel to the vector $\vec{R}_{3}$ and that $\vec{R}_{2}, \vec{e}_{x} \mathrm{BF}$ and $\vec{e}_{z} \mathrm{BF}$ lie in the same half-plane (see Fig. 1).

It has been shown elsewhere ${ }^{39-42}$ that polyspherical coordinates lead to general and compact forms of kinetic energy operators. The explicit expression of the present kinetic energy operator (KEO) is given in Appendix A. The correctness of its derivation was checked by comparing it with numerical results provided by the program TNUM. ${ }^{43}$ The KEO can be formally written as $\hat{T}=(1 / 2) \sum_{i j} \partial_{i} G_{i j}(\mathbf{q}) \partial_{j}+V_{\text {extra }}(\mathbf{q})$. TNUM computes $G$ and $V_{\text {extra }}$ numerically on a grid. We have checked that the numerical values of all the functions $G_{i j}(\mathbf{q})$ and $V_{\text {extra }}(\mathbf{q})$ at several non-symmetrical grid points, $\mathbf{q}$, agree with those provided by the program TNUM. ${ }^{43}$ The implementation of this KEO, including the overall rotation for a tetra-atomic molecule in non-orthogonal coordinates, in the Heidelberg MCTDH package is an important result of the present work, since this operator could be useful for many systems in the future.

\section{B. Model}

As in ref. 20, we use a time-dependent Hamiltonian. In particular, we use the Loudon ${ }^{44}$ presentation that describes the molecule quantum mechanically, the electric field classically and their interaction by first order perturbation theory, i.e.

$$
\hat{H}_{\text {tot }}(t)=\hat{H}_{\mathrm{o}}+\hat{h}(t),
$$

$\hat{H}_{\mathrm{o}}$ is the Hamiltonian of HONO without external field.

$$
\hat{H}_{o}=\hat{T}+\hat{V},
$$

where $\hat{T}$ is the exact kinetic energy operator including the overall rotation and Coriolis couplings given in Appendix A. $\hat{V}$ is the six dimensional potential energy surface calculated in ref. 18. $\hat{h}(t)$ denotes the interaction of the molecule with a classical external laser field $E(t),{ }^{44}$ i.e.

$$
\hat{h}(t)=-\hat{\vec{\mu}} \cdot \vec{E}(t),
$$

where $\hat{\vec{\mu}}$ is the dipole moment operator calculated in ref. 20. As in ref. 20, we adopt the following form for the external field:

$$
\vec{E}(t)=E_{0} \cos (\omega t) \sin ^{2}\left(\pi t / t_{\mathrm{p}}\right) \Theta(t) \Theta\left(t_{\mathrm{p}}-t\right) \vec{e},
$$

where $E_{0}$ is the field strength, $\omega$ the field frequency, $\Theta(t)$ the Heavyside's step function, $t_{\mathrm{p}}$ the pulse duration, and $\vec{e}$ the unit vector in the polarization direction.

In ref. 20, the six-dimensional Dipole Moment Surface (DMS) was generated by ab initio calculations using the density functional theory approach with the B97-1 functional ${ }^{45}$ implemented in CADPAC. ${ }^{46}$ The components of the dipole moment vector have been fitted to the following form:

$\mu_{x}\left(R_{1}, R_{2}, R_{3}, \theta_{1}, \theta_{2}, \tau\right)=\sum_{i j k l m n} c_{i j k l m n}^{x} R_{1}^{i} R_{2}^{j} R_{3}^{k} \theta_{1}^{l} \theta_{2}^{m} \cos (n \tau)$

with restriction given in ref. 20 .

In ref. 20, we have determined an efficient selective IVR-pathway leading to the cis $\rightarrow$ trans isomerization. This path corresponds to a simultaneous increase of the (middle) $\mathrm{ON}$ stretch and of the ONO bending. We have observed that the selective IVR-pathway corresponds to an excitation of the 
$010 a 00 / 000(a+2) 00$ series of eigenstates (see ref. 20 for the definition of the normal and local modes). We have simulated the dynamics with MCTDH and in the presence of a laser pulse with a carrier frequency of $850 \mathrm{~cm}^{-1}$, an intensity of 0.0035 a.u., and an irradiation duration of $500 \mathrm{fs}$. We have predicted that HONO isomerizes in the gas phase with a yield of about 10 percent when the suggested parameters for the pulse are used. Higher yields are likely if higher intensities or longer irradiation times are used.

In ref. 20, the initial wave function was the vibrational ground state (for $J=0$ ). In the present work, we take the same parameters for the pulse but use the rovibrational eigenstates calculated in Chapter 3 to define the initial wave functions. This allows us to focus on the effect of the rotation on the isomerization described in ref. 20. In addition, the field is oriented parallel to the $z^{B F}$ axis, i.e. we consider the effect of a projection of the external field onto this axis only. Indeed, as discussed in ref. 20, we found no isomerization for $x$ and $y$ polarizations and a laser pulse with a carrier frequency of $850 \mathrm{~cm}^{-1}$ and a field strength of 0.0035 a.u. $\left(4.3 \times 10^{11}\right.$ Watt $\mathrm{cm}^{-2}$ ). An orientation of $45^{\circ}$ between the $x$ and $z$ axes yielded about half of the final isomerization in the $z$ polarized case. Thus, assuming isotropic orientation during the laser irradiation, we concluded that only one third of the calculated isomerization would be observed. Now, choosing an external field parallel to the $\mathrm{ON}$ bond in the simulations amounts to focusing on the action of the projection of an external field parallel to a laboratory fixed axis onto the $z^{\mathrm{BF}}$ axis of the molecule only. In a future work, we will investigate the direct excitation of the overall rotation (i.e. transitions between $J$ and $J^{\prime}$ with $J^{\prime} \neq J$ ) by the laser pulse by starting from an external field parallel to a Laboratory-Fixed frame and including the Euler rotation matrix in the scalar product, $-\hat{\vec{\mu}} \cdot \vec{E}(t)$. This will allow transitions between $J$ and $J^{\prime}$ with $J^{\prime} \neq J$. But it is not the topic of the present work where we focus on the robustness of the chemical process studied ${ }^{20}$ with respect to overall rotation.

As in our former studies, the Heidelberg package ${ }^{8}$ of the Multi-Configuration Time Dependent Hartree (MCTDH) algorithm $^{9,10,12}$ is employed to simulate the dynamics.

The parameters of the grids used are provided in Table 1 and the sizes of the single-particle functions basis sets are given in Table 2.

\section{Rovibrational eigenfunctions}

In the present section, we calculate the $7 \mathrm{D}$ rovibrational eigenstates corresponding to the vibrational ground state and for the cis geometry. These eigenstates will be used as initial wave functions for the dynamics of HONO in Section V. The corresponding eigenvalues are compared with the
Table 2 Number of SPFs used for the four modes in the MCTDH calculations. Four modes (MCTDH particles) are defined in the MCTDH-calculations: $\left(\mathrm{R}_{1}, \varphi\right),\left(R_{3}, \cos \theta_{2}\right),\left(R_{2}, \cos \theta_{1}\right)$, and $k$, where the brackets indicate the mode combinations. The spf basis set for the "Pulse (150 15)" is the one used for the dynamics starting from the eigenstate denoted $\left(\begin{array}{lll}15 & 0 & 15\end{array}\right)$ in Table 5 . The spf basis set for the Relaxation $(550)$ is the one used to converge the eigenstate denoted (5 50 ) in Table 3

\begin{tabular}{lllll}
\hline & $\left(R_{1}, \varphi\right)$ & $\left(R_{3}, \cos \theta_{2}\right)$ & $\left(R_{2}, \cos \theta_{1}\right)$ & $k$ \\
\hline Pulse (15 0 15) & 35 & 45 & 35 & 20 \\
Relaxation (5 50) & 40 & 45 & 40 & 11 \\
\hline
\end{tabular}

experimental data given in ref. 27. We used the "improved relaxation" method ${ }^{11,17,48}$ to obtain the 7D vibrational eigenenergies and eigenfunctions of $\hat{H}_{0}$. Some of the eigenvalues for the vibrational ground state with $J=5,10,15$, and 22 are given in Tables 3-5. The experimental values taken from ref. 27 are also provided.

In order to label the rovibrational eigenstates, we use a rigid rotor approximation as a zero-order description of the rotation of the system. One knows that an asymmetric rigid body can be characterized by the three eigenvalues of the moment of inertia tensor, which are real-valued and positive numbers $\left(I_{a}, I_{b}, I_{c}\right)$, known as principal moments of inertia. The corresponding kinetic energy operator reads ${ }^{49}$

$$
\hat{H}_{\mathrm{rot}}=A J_{a}^{2}+B J_{b}^{2}+C J_{c}^{2},
$$

where $A, B$ and $C$ are the principal rotational constants:

$$
A=\frac{1}{2 I_{a}}, \quad B=\frac{1}{2 I_{b}}, \quad C=\frac{1}{2 I_{c}},
$$

with $A>B>C$. The convention $\hbar=1$ is used everywhere in the present paper. $J_{a}, J_{b}$, and $J_{c}$ are the projections of the total angular momentum onto the principal axes of inertia, $a, b, c$. We use the notation $k_{a}, k_{b}$ and $k_{c}$ for the corresponding quantum number. In the case of a prolate symmetric rotor, we have

$$
I_{c}=I_{b}>I_{a} \text {, i.e. } A>B=C,
$$

with

$$
\hat{H}=B \hat{J}^{2}+(A-B) \hat{J}_{a}^{2} .
$$

The corresponding eigenvalues are given as

$$
E_{J, k_{a}}=B J(J+1)+(A-B) k_{a}^{2} .
$$

In the case of an oblate symmetric rotor $I_{c}>I_{b}=I_{a}$, we have

$$
\hat{H}=B \hat{J}^{2}+(C-B) \hat{J}_{c}^{2}
$$

\begin{tabular}{|c|c|c|c|c|c|c|c|}
\hline & $\left(R_{1}\right.$ & $\tau)$ & $\left(R_{3}\right.$ & $\left.\cos \theta_{2}\right)$ & $\left(R_{2}\right.$ & $\left.\cos \theta_{1}\right)$ & $k$ \\
\hline Primitive basis & HO-DVR & exp-DVR & HO-DVR & HO-DVR & HO-DVR & HO-DVR & $k$ \\
\hline $\begin{array}{l}\text { Basis } \\
\text { Grid length/a.u. }\end{array}$ & $\begin{array}{l}20 \\
{[1.3,2.5]}\end{array}$ & $\begin{array}{l}69 \\
{[0,2 \pi]}\end{array}$ & $\begin{array}{l}45 \\
{[1.5,4.0]}\end{array}$ & $\begin{array}{l}30 \\
{[-0.9,0.1]}\end{array}$ & $\begin{array}{l}20 \\
{[1.8,2.7]}\end{array}$ & $\begin{array}{l}35 \\
{[-0.95,0.4]}\end{array}$ & $\begin{array}{l}2 J+1 \\
{[-J,+J]}\end{array}$ \\
\hline
\end{tabular}

with

$$
E_{J, k_{c}}=B J(J+1)+(C-B) k_{c}^{2}
$$

Table 1 Parameters for the primitive basis set employed for all the calculations. HO denotes a harmonic oscillator (Hermite) discrete variable representation (DVR) 
Table 3 Comparison of calculated and experimental rovibrational

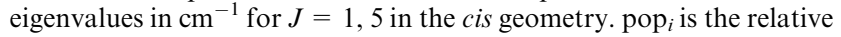
population with respect to the ground state given by eqn (15). $k_{a}^{\prime}$ and $k_{c}^{\prime}$ are the quantum numbers proposed by the experimentalists ${ }^{27}$ to label the eigenstates. Sym. denotes the symmetry $\mathrm{A}^{\prime}$ or $\mathrm{A}^{\prime \prime}$ of the $7 \mathrm{D}$ eigenstates

\begin{tabular}{|c|c|c|c|c|c|}
\hline$\left(\begin{array}{lll}J & k_{a}^{\prime} & k_{c}^{\prime}\end{array}\right)$ & obs. ${ }^{a}$ & $E_{\mathrm{MCTDH}}{ }^{b}$ & Sym. & $\frac{100 \text { pop }_{i}}{\text { pop }_{J}}(298 \mathrm{~K})^{c}$ & $\frac{100 \text { pop }_{i}}{\text { pop }_{J}}(10 \mathrm{~K})^{c}$ \\
\hline $\begin{array}{lll}10 & 1\end{array}$ & 0.82 & 0.81 & $\mathrm{~A}^{\prime}$ & 33.6 & 41.4 \\
\hline 111 & 3.18 & 3.17 & $\mathrm{~A}^{\prime}$ & 33.2 & 29.4 \\
\hline 110 & 3.24 & 3.23 & $\mathrm{~A}^{\prime \prime}$ & 33.2 & 29.2 \\
\hline 505 & 12.24 & 12.18 & $\mathrm{~A}^{\prime}$ & 10.2 & 33.2 \\
\hline 515 & 14.21 & 14.15 & $\mathrm{~A}^{\prime}$ & 10.1 & 25.0 \\
\hline 514 & 15.11 & 15.05 & $\mathrm{~A}^{\prime \prime}$ & 10.0 & 21.9 \\
\hline 524 & 21.85 & 21.76 & $\mathrm{~A}^{\prime \prime}$ & 9.69 & 8.35 \\
\hline 523 & 21.89 & 21.80 & $\mathrm{~A}^{\prime}$ & 9.69 & 8.31 \\
\hline 533 & 33.84 & 33.70 & $\mathrm{~A}^{\prime}$ & 9.15 & 1.50 \\
\hline 532 & 33.84 & 33.70 & $\mathrm{~A}^{\prime \prime}$ & 9.15 & 1.50 \\
\hline 542 & 50.60 & 50.39 & $\mathrm{~A}^{\prime \prime}$ & 8.44 & 0.14 \\
\hline 541 & 50.60 & 50.39 & $\mathrm{~A}^{\prime}$ & 8.44 & 0.14 \\
\hline 551 & 72.14 & 71.84 & $\mathrm{~A}^{\prime}$ & 7.61 & 0.01 \\
\hline 550 & 72.14 & 71.84 & $\mathrm{~A}^{\prime \prime}$ & 7.61 & 0.01 \\
\hline
\end{tabular}

${ }^{a}$ Experimental excitation energies taken from ref. 27. ${ }^{b}$ This work using the improved relaxation method. ${ }^{c}$ Relative population of the $i$ th state with respect to the ground state evaluated with eqn (15).

In the particular case of HONO in its cis geometry, we obtained in ref. 18:

$$
A=2.792 \mathrm{~cm}^{-1}, B=0.438 \mathrm{~cm}^{-1}, C=0.378 \mathrm{~cm}^{-1} \text {. }
$$

Since the "asymmetry parameter",

$$
\kappa_{R}=\frac{2 B-A-C}{A-C},
$$

is equal to -0.950 for $\mathrm{HONO}$, i.e. close to -1 , we can describe the rotation of $\mathrm{HONO}$, in a first order description, by a prolate symmetric rotor, i.e. in the low energy domain $k_{a}$ is almost a good quantum number but $k_{c}$ is not. However, $k_{c}$ is linked to the parity of the eigenstates. The values $k_{a}^{\prime}$ and $k_{c}^{\prime}$ given in Tables $3-5$ are the values of $\left\langle\hat{J}_{a}\right\rangle$ and $\left\langle\hat{J}_{c}\right\rangle$ proposed by the experimentalists ${ }^{27}$ to label their measured eigenvalues.

Tables 3-5 reveal an excellent agreement with the experimental values taken from Guilmot et al., ${ }^{27}$ which confirms the high quality of the potential surface and the reliability of the approach adopted in the present study. This is an important result of the present work: in ref. 20, the comparison with the experimental vibrational eigenvalues and the transition moments was also excellent. In addition, Fig. 2-4 show the one-dimensional densities of eigenstates of several rovibrational eigenstates along $k$, where $k$ denotes the quantum number associated with the projection of the total angular momentum onto the $z^{\mathrm{BF}}$ axis, $\hat{J}_{z_{\mathrm{BF}}}$. For these eigenstates, the total angular momentum, $J$, is set to 1,5 , or 10 .

Fig. 2-4 reveal a very complex structure: almost all the values of $k$ are populated. It must be emphasized that $k$ is different from $k_{a}$ because we are not using an Eckart frame (ref. 47) and that the distribution along $k$ is much more complex than the one along $k_{a}$.

Finally, in order to estimate the relative populations of the HONO molecules occupying the rovibrational states at a
Table 4 Comparison of calculated and experimental rovibrational eigenvalues in $\mathrm{cm}^{-1}$ for $J=10$ and the cis geometry. pop p $_{i}$ is the relative population with respect to the ground state given by eqn (15). $k_{a}^{\prime}$ and $k_{c}^{\prime}$ are the quantum numbers given by the experimentalists ${ }^{27}$ to label the eigenstates. Sym. denotes the symmetry $\mathrm{A}^{\prime}$ or $\mathrm{A}^{\prime \prime}$ of the $7 \mathrm{D}$ eigenstates

\begin{tabular}{lllllll}
$\left(J k_{a}^{\prime} k_{c}^{\prime}\right)$ & obs. $^{a}$ & $E_{\mathrm{MCTDH}^{b}}$ & Sym. & $\frac{100 \text { pop }_{i}}{\text { pop }_{J}}(298 \mathrm{~K})^{c}$ & $\frac{100 \text { pop }_{i}}{\text { pop }_{J}}(10 \mathrm{~K})^{c}$ \\
\hline 10010 & 44.47 & 44.28 & $\mathrm{~A}^{\prime}$ & 6.82 & 34.2 \\
10110 & 45.62 & 45.43 & $\mathrm{~A}^{\prime}$ & 6.79 & 29.0 \\
1019 & 48.91 & 48.70 & $\mathrm{~A}^{\prime \prime}$ & 6.68 & 18.1 \\
1029 & 54.51 & 54.28 & $\mathrm{~A}^{\prime \prime}$ & 6.50 & 8.11 \\
1028 & 55.04 & 54.81 & $\mathrm{~A}^{\prime}$ & 6.49 & 7.51 \\
1038 & 66.63 & 66.35 & $\mathrm{~A}^{\prime}$ & 6.13 & 1.43 \\
1037 & 66.65 & 66.37 & $\mathrm{~A}^{\prime \prime}$ & 6.13 & 1.43 \\
1047 & 83.37 & 83.02 & $\mathrm{~A}^{\prime \prime}$ & 5.66 & 0.13 \\
1046 & 83.37 & 83.02 & $\mathrm{~A}^{\prime}$ & 5.66 & 0.13 \\
1056 & 104.9 & 104.46 & $\mathrm{~A}^{\prime}$ & 5.10 & 0.01 \\
1055 & 104.9 & 104.46 & $\mathrm{~A}^{\prime \prime}$ & 5.10 & 0.01 \\
1065 & 131.2 & 130.68 & $\mathrm{~A}^{\prime \prime}$ & 4.50 & 0 \\
1064 & 131.2 & 130.68 & $\mathrm{~A}^{\prime}$ & 4.50 & 0 \\
1074 & 162.27 & 161.63 & $\mathrm{~A}^{\prime}$ & 3.87 & 0 \\
1073 & 162.27 & 161.63 & $\mathrm{~A}^{\prime \prime}$ & 3.87 & 0 \\
1083 & 198.1 & 197.31 & $\mathrm{~A}^{\prime \prime}$ & 3.26 & 0 \\
1082 & 198.1 & 197.31 & $\mathrm{~A}^{\prime}$ & 3.26 & 0 \\
1092 & 238.67 & 237.71 & $\mathrm{~A}^{\prime}$ & 2.68 & 0 \\
1091 & 238.67 & 237.71 & $\mathrm{~A}^{\prime \prime}$ & 2.68 & 0 \\
10101 & 283.97 & 282.83 & $\mathrm{~A}^{\prime \prime}$ & 2.16 & 0 \\
1010 & 0 & 283.97 & 282.83 & $\mathrm{~A}^{\prime}$ & 2.16 & 0
\end{tabular}

${ }^{a}$ Experimental excitation energies taken from ref. 27. ${ }^{b}$ This work using the improved relaxation method. ${ }^{c}$ Relative population of the $i$ th state with respect to the ground state evaluated with eqn (15).

Table 5 Comparison of selected, calculated and experimental rovibrational eigenvalues in $\mathrm{cm}^{-1}$ for $J=12,15,22$ in the cis geometry. $\operatorname{pop}_{i}$ is the relative population with respect to the ground state given by eqn (15). $k_{a}^{\prime}$ and $k_{c}^{\prime}$ are the quantum numbers given by the experimentalists ${ }^{27}$ to label the eigenstates. Sym. denotes the

\begin{tabular}{|c|c|c|c|c|c|}
\hline$\left(J k_{a}^{\prime} k_{c}^{\prime}\right)$ & obs. ${ }^{a}$ & $E_{\text {MCTDH }}^{b}$ & Sym. & $\frac{100 \text { pop }_{i}}{\text { pop }_{J}}(298 \mathrm{~K})^{c}$ & $\frac{100 \text { pop }_{i}}{\text { pop }_{J}}(10 \mathrm{~K})^{c}$ \\
\hline 12012 & 62.78 & 62.49 & $\mathrm{~A}^{\prime}$ & 6.45 & 35.2 \\
\hline 12112 & 63.63 & 63.33 & $\mathrm{~A}^{\prime}$ & 6.43 & 31.2 \\
\hline 12111 & 68.25 & 67.94 & $\mathrm{~A}^{\prime \prime}$ & 6.29 & 16.0 \\
\hline 12211 & 73.23 & 72.92 & $\mathrm{~A}^{\prime \prime}$ & 6.14 & 7.87 \\
\hline 12210 & 74.26 & 73.95 & $\mathrm{~A}^{\prime}$ & 6.11 & 6.79 \\
\hline 12121 & 407.53 & 405.86 & $\mathrm{~A}^{\prime \prime}$ & 1.22 & 0 \\
\hline 12120 & 407.53 & 405.86 & $\mathrm{~A}^{\prime}$ & 1.22 & 0 \\
\hline 15015 & 95.93 & 95.52 & $\mathrm{~A}^{\prime}$ & 6.23 & 37.3 \\
\hline 15115 & 96.42 & 96.00 & $\mathrm{~A}^{\prime}$ & 6.22 & 34.8 \\
\hline 15151 & 634.07 & 631.46 & $\mathrm{~A}^{\prime}$ & 0.46 & 0 \\
\hline 15150 & 634.07 & 631.46 & $\mathrm{~A}^{\prime \prime}$ & 0.46 & 0 \\
\hline 22022 & 199.57 & 198.72 & $\mathrm{~A}^{\prime}$ & 6.27 & 43.2 \\
\hline 22122 & 199.68 & 198.83 & $\mathrm{~A}^{\prime}$ & 6.27 & 42.6 \\
\hline 22121 & 212.97 & 212.07 & $\mathrm{~A}^{\prime \prime}$ & 5.88 & 6.30 \\
\hline 22221 & - & 1346.40 & $\mathrm{~A}^{\prime \prime}$ & 0.02 & 0 \\
\hline 22220 & - & 1346.40 & $\mathrm{~A}^{\prime}$ & 0.02 & 0 \\
\hline
\end{tabular}
symmetry $\mathrm{A}^{\prime}$ or $\mathrm{A}^{\prime \prime}$ of the $7 \mathrm{D}$ eigenstates.

${ }^{a}$ Experimental excitation energies taken from ref. 27. ${ }^{b}$ This work using the improved relaxation method. ${ }^{c}$ Relative population of the $i$ th state with respect to the ground state evaluated with eqn (15).

temperature $T$, we used the Boltzmann distribution law, which states that these relative populations are given as

$$
\operatorname{pop}_{i}=\frac{N_{i}}{N_{0}}=\frac{g_{i}}{g_{0}} \exp \left(\frac{-\Delta E_{i}}{k_{\mathrm{B}} T}\right),
$$



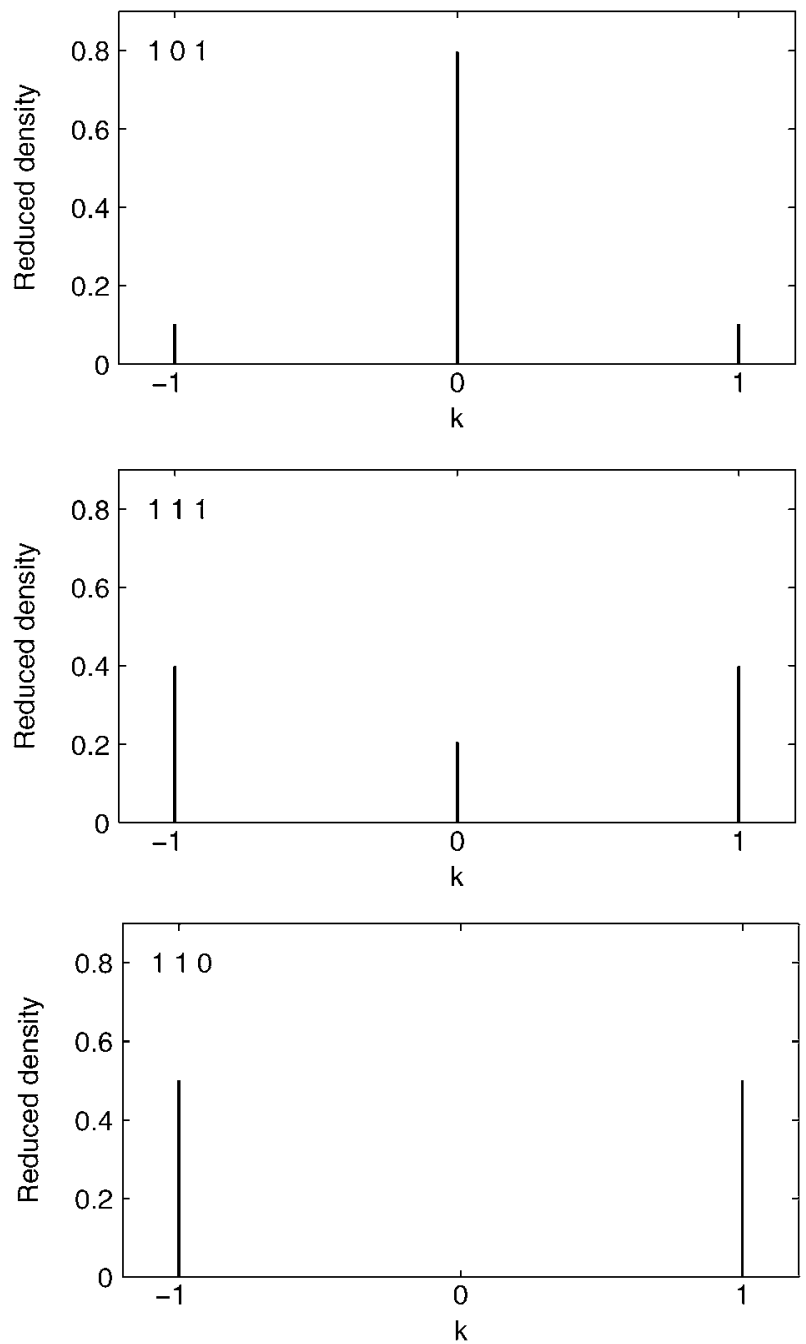

Fig. 2 One-dimensional probability distributions of $k$ for eigenstates $(1,0,1),(1,1,1)$, and $(1,1,0)$ (see Table 3$). k$ is the projection of the total angular momentum onto the $z^{\mathrm{BF}}$ axis as defined in Fig. 1 and not the projection, $k_{a}$, onto the principal axis. The one-dimensional probability distribution is obtained by integrating the square of the wave function over the six internal degrees of freedom. This probability is independent of temperature.

where $N_{i}$ and $N_{0}$ are the number of molecules in the $i$ th and the ground states, respectively. $\Delta E_{i}=E_{i}-E_{0}$, with $E_{i}$ and $E_{0}$ being the energies of these states. $k_{\mathrm{B}}$ is Boltzmann's constant and $g_{i}$ and $g_{0}$ are the degeneracies of the $i$ th and the ground states, respectively. Here, we have $g_{i}=(2 J+1)$ since there are $2 J+1$ values of the projection of the total angular momentum onto the $z^{\mathrm{SF}}$ axis and $g_{0}=1$. We took $T=10$ and 298 Kelvin. $100 * \mathrm{pop}_{i} / \operatorname{pop}_{J}$ is provided for all the calculated eigenstates in Tables 3-5. The population for a given $J$, pop $J$, is given as

$$
\begin{aligned}
\operatorname{pop}_{J} & =\sum_{k_{a}=-J, \ldots,+J} \frac{N_{k_{a}}}{N_{0}} \\
& =\sum_{k_{a}=-J, \ldots,+J}(2 J+1) \exp \left(\frac{-\Delta E_{k_{a}}}{k_{\mathrm{B}} T}\right),
\end{aligned}
$$

where $k_{a}$ runs over the $(2 J+1)$ eigenstates associated with quantum number $J$. According to our calculations, the maximum of pop $_{J}$ is reached for $J=16$ at room temperature and for $J=3$ at $T=10 \mathrm{~K}$.

It must be stressed that the reference to the rigid rotor of HONO is important only to label the initial eigenstates that we will use for the dynamics of HONO and for the comparison with the experimental eigenvalues. As explained in ref. 20, the laser pulse we use creates a superposition of many eigenstates (see Fig. 4 of ref. 20). Those that lead to the isomerization lie $4000 \mathrm{~cm}^{-1}$ above the zero point energy.

\section{Symmetry considerations}

For floppy systems exhibiting several minima, it is necessary to work with the complete nuclear-permutation-inversion (CNPI) group (as opposed to the point group of symmetry of any given geometry). ${ }^{50}$ In the case of HONO, the exchange of oxygen atoms is considered unfeasible. Thus, in the process studied here, the nuclear-permutation-inversion group turns into the $C_{s}(\mathrm{M})$ group (see Table A-2, page 670 in ref. 50). $C_{s}(\mathrm{M})$ contains identity and inversion only.

The effect of the inversion operation, $E^{*}$, on the polyspherical coordinates is given by

$$
E^{*}\left[\begin{array}{c}
\alpha \\
\beta \\
\gamma \\
\tau
\end{array}\right]=\left[\begin{array}{c}
\pi+\alpha \\
\pi-\beta \\
-\gamma \\
-\tau
\end{array}\right] .
$$

The other coordinates are not affected. The angular basis set that describes the overall rotation, parametrized by three Euler angles, reads ${ }^{51}$

$$
D_{M k}^{J}{ }^{*}(\alpha, \beta, \gamma)=\mathrm{e}^{i M \alpha} d_{M \Omega}^{J}(\beta) \mathrm{e}^{i k \gamma},
$$

where $D_{M k}^{J}{ }^{*}(\alpha, \beta, \gamma)$ and $d_{M k}^{J}(\beta)$ denote the Wigner matrix elements and the Jacobi functions, respectively. $M$ is the projection of the total angular momentum onto the $z^{\mathrm{SF}}$ axis. In addition, eqn (3.67), (3.68) and (3.75) of ref. 51 yield:

$$
\begin{aligned}
d_{M k}^{J}(\pi-\beta) & =(-1)^{k+J} d_{M-k}^{J}(-\beta) \\
& =(-1)^{k+J}(-1)^{k+M} d_{M-k}^{J}(\beta) \\
& =(-1)^{J+M} d_{M-k}^{J}(\beta) .
\end{aligned}
$$

Now, starting from the following basis set for the three Euler angles and $\tau$

$$
D_{M k}^{J} *(\alpha, \beta, \gamma) \mathrm{e}^{i \nu \tau},
$$

we have

$$
D_{M k}^{J}{ }^{*}(\pi+\alpha, \pi-\beta,-\gamma) \mathrm{e}^{-i \nu \tau}=(-1)^{J}\left(D_{M-k}^{J}{ }^{*}(\alpha, \beta, \gamma) \mathrm{e}^{-i \nu \tau}\right),
$$

The $\mathrm{A}_{1}{ }^{\prime}$ symmetrized basis set is thus given by

$$
\left(D_{M k}^{J}{ }^{*}(\alpha, \beta, \gamma) \mathrm{e}^{i \nu \tau}\right)+(-1)^{J}\left(D_{M-k}^{J}{ }^{*}(\alpha, \beta, \gamma) \mathrm{e}^{-i \nu \tau}\right),
$$

and the $\mathrm{A}_{1}{ }^{\prime \prime}$ symmetrized basis set is given by

$$
\left(D_{M k}^{J}{ }^{*}(\alpha, \beta, \gamma) \mathrm{e}^{i \nu \tau}\right)-(-1)^{J}\left(D_{M-k}^{J}{ }^{*}(\alpha, \beta, \gamma) \mathrm{e}^{-i \nu \tau}\right) .
$$

As usual with MCTDH, we do not take into account this symmetry explicitly when building the primitive basis. However, the previous equations are very important to determine the symmetry of the eigenstates converged with MCTDH. For 

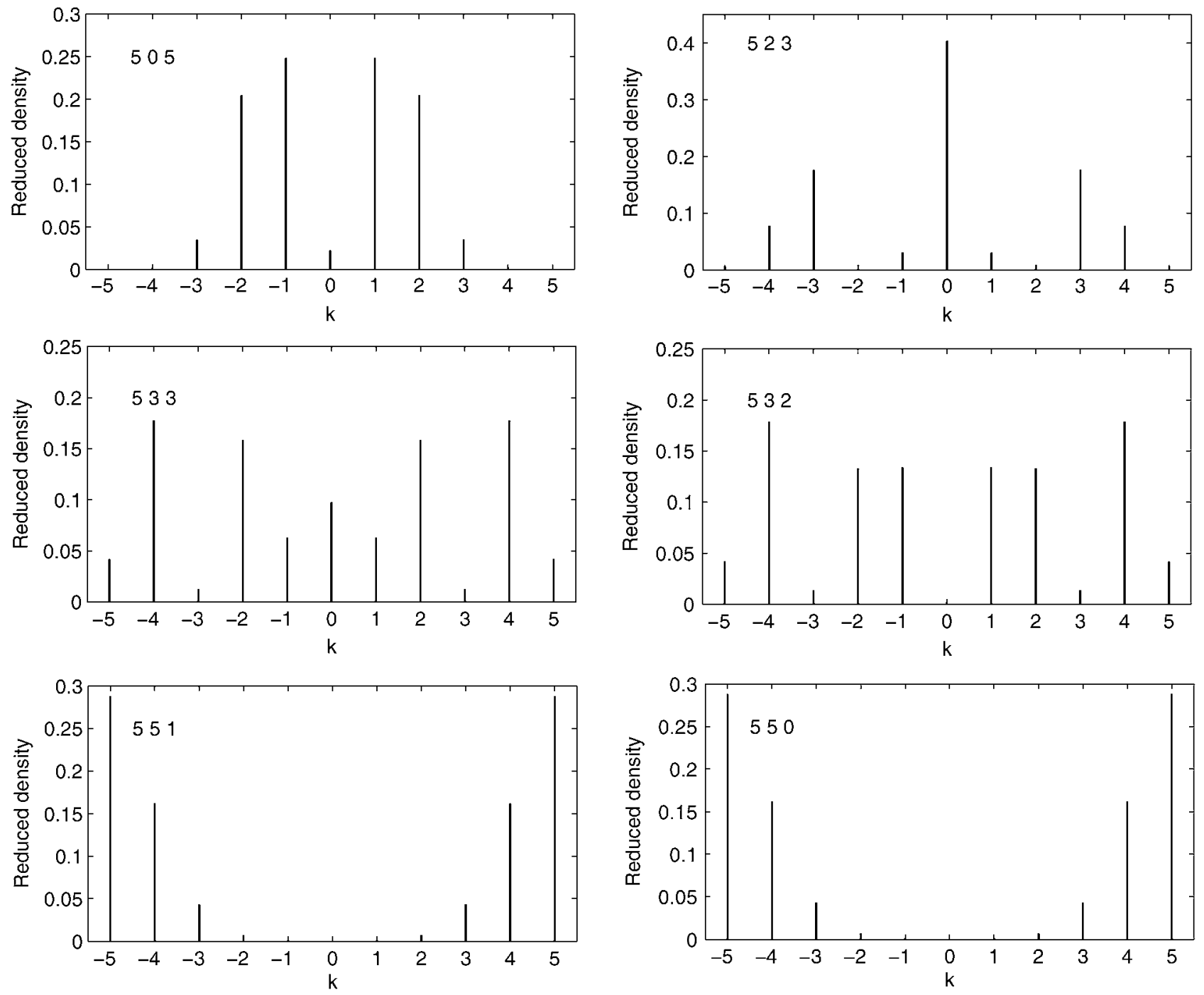

Fig. 3 Similar to Fig. 2 but $k$ for eigenstates $(5,0,5),(5,2,3),(5,3,3),(5,3,2),(5,5,1)$ and $(5,5,0)$ (see Table 3).

non-degenerate eigenstates, the improved relaxation approach used in Section III always yields the eigenstates with correct symmetry. The $\mathrm{A}^{\prime}$ and $\mathrm{A}^{\prime \prime}$ character of the eigenstates can then be determined by visual inspection of the corresponding wave functions: since for $\nu=0$, the distribution in the quantum number $\nu$ is symmetric, one just has to look at the even or odd character of the distribution in $k$.

However, for HONO and $\nu=0$, some eigenstates are structurally degenerate. For those eigenstates, we have observed that the improved relaxation does not provide the two eigenstates with the $\mathrm{A}^{\prime}$ and $\mathrm{A}^{\prime \prime}$ symmetries but rather their sum and their difference. We have thus resymmetrized the two eigenvectors by taking their sum and their difference. The symmetry of the eigenstates used as initial wave functions for the propagations is provided in Tables 3-5. In these tables, we also give the labels of the eigenstates given by the experimentalists. Their labels are based on the rigid rotor description detailed in Section III: $k_{a}^{\prime}$ and $k_{c}^{\prime}$ are the projections of the total angular momentum onto the $a$ and $c$ principal axes. Again, we stress that $k_{a}^{\prime}$ is different from our $k$ since $k$ corresponds to the projection of the total angular momentum onto $z^{\mathrm{BF}}$ with the definition of the $\mathrm{BF}$ frame given in Section IIA. When using a rigid rotor description, the parity of the rotational states is given by $(-1)^{J-k_{c}^{\prime}}$. However, it must be emphasized that the symmetry conditions used here are given by eqn (22) and (23) and do not involve $k_{a}^{\prime}$ and $k_{c}^{\prime}$ since our conditions are valid for any distorted geometry and not for the rigid rotor only. As explained in ref. 50, the use of the nuclear-permutation-inversion (CNPI) group is the only way to generalize the point group of symmetry for vibrations around the equilibrium geometry (see also ref. 52 and 53 for a systematic combination of polyspherical coordinates and nuclear-permutation-inversion group).

\section{Influence of the overall rotation on the excitation of HONO by a laser pulse}

The kinetics of the isomerization is governed by the time evolution of the population of the trans isomer, $P_{\text {trans }}$. Its time evolution is obtained by projecting the time dependent wave packet onto the interval $\pi / 2 \leq \tau \leq 3 \pi / 2$

$$
P_{\text {trans }}=\langle\Psi|\Theta(\tau-\pi / 2) \Theta(3 \pi / 2-\tau)| \Psi\rangle
$$



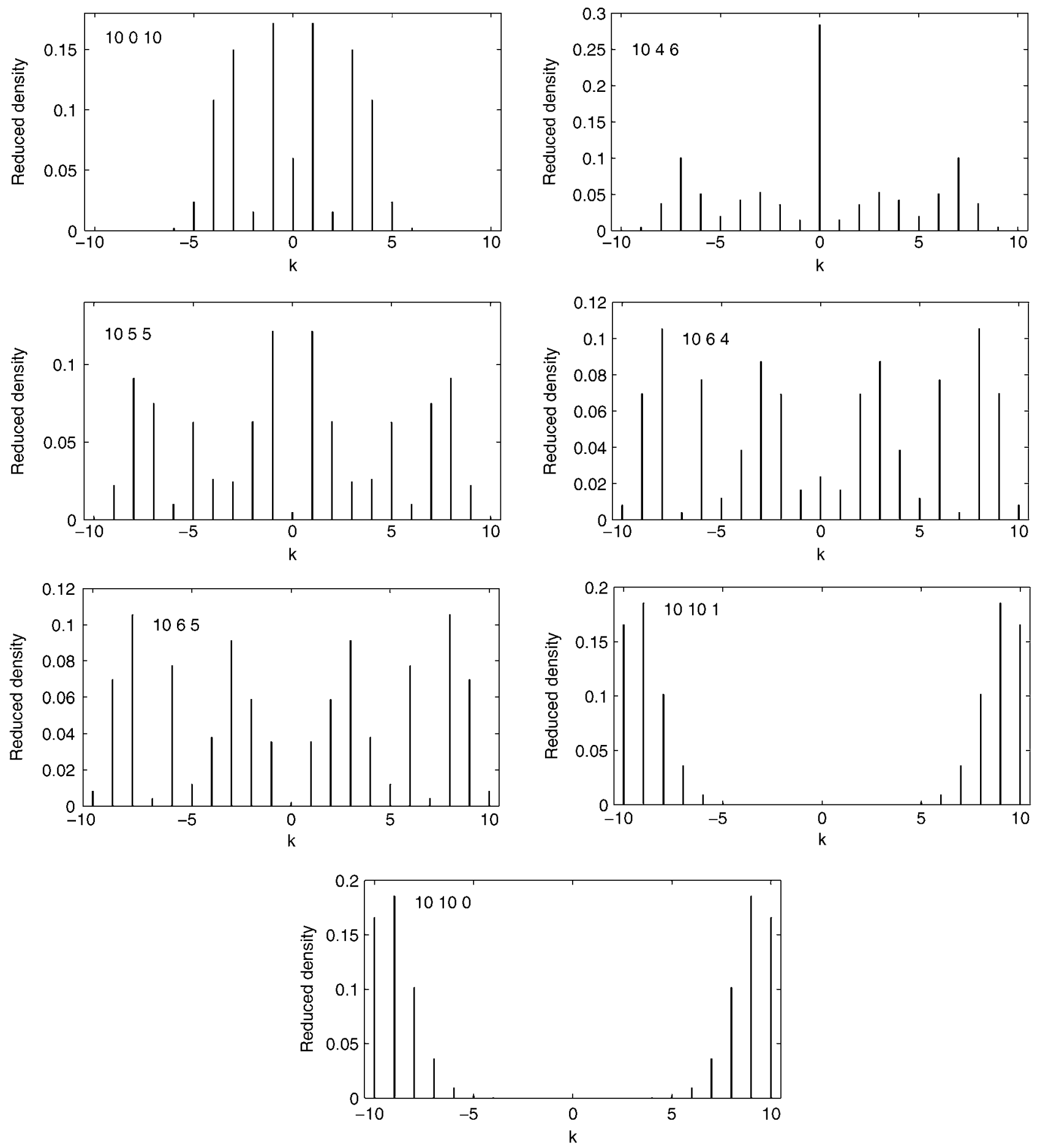

Fig. 4 Similar to Fig. 2 but $k$ for eigenstates $(10,0,10),(10,4,6),(10,5,5),(10,6,4),(10,6,5),(10,10,1)$ and $(10,10,0)($ see Table 5).

Fig. 5 shows the comparison of $P_{\text {trans }}$ obtained with $J=0$ with the three initial states corresponding to the three eigenstates for $J=1$.

The dynamics starting from $(1,1,0),(1,0,1)$, and $(1,1,1)$ shows a little bit more isomerization than for the case with $J=0$ even during the pulse excitation (here, we use the notation $\left(k_{a}^{\prime}, k_{b}^{\prime}, k_{c}^{\prime}\right)$ to label the eigenstates). The shape of the time evolution of $P_{\text {trans }}$ does not change much. Even though $(1,0,1)$ and $(1,1,1)$ have almost the same energy, the shapes of the time evolution of $P_{\text {trans }}$ when starting from these two initial states are slightly different due to the fact that they have a different symmetry $\left(\mathrm{A}^{\prime}\right.$ for $(1,1,1)$ and $\mathrm{A}^{\prime \prime}$ for $\left.(1,1,0)\right)$. Indeed, for each value of $J$, except for the first initial eigenstate $(J, 0, J)$, all the eigenstates can be associated by groups of two states: one with the $\mathrm{A}^{\prime}$ symmetry, the other with the $\mathrm{A}^{\prime \prime}$ symmetry. When $k_{a}^{\prime}$ is small, the two corresponding eigenvalues are close to one another. When $k_{a}^{\prime}$ increases, the two eigenstates structurally accidentally degenerate. In addition, when $k_{a}^{\prime}$ 


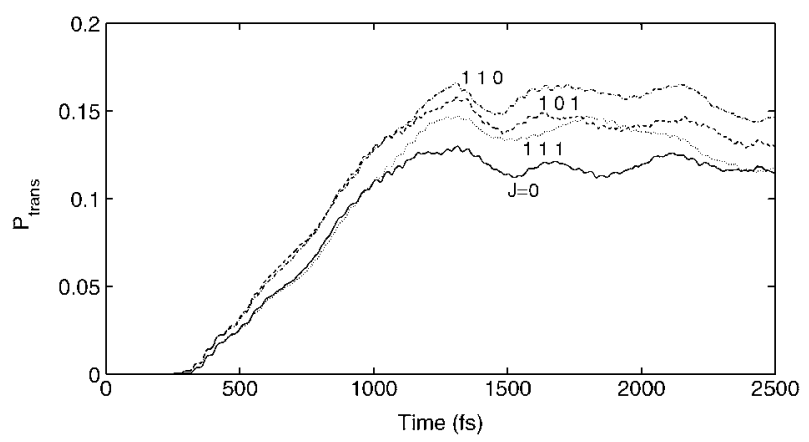

Fig. 5 Time evolution (during and after excitation) of $P_{\text {trans }}$ (see eqn (24) in the text). The pulse is on during the interval 0-500 fs: see eqn (4). The solid line denotes the time evolution for $J=0$ (taken from ref. 20). Dashed lines correspond to the time evolutions of $P_{\text {trans }}$ starting from the three rovibrational eigenstates for $J=1$ with the labels of Table 3.

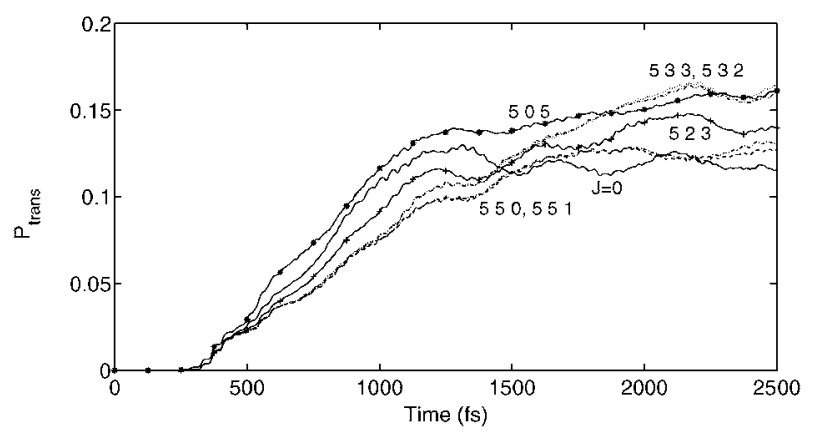

Fig. 6 Similar to Fig. 5 but for $J=5$ with the labels of Table 3 .

increases for a given $J$, the time evolutions of $P_{\text {trans }}$ starting from the two eigenstates become closer and closer. For instance, the shapes of the time evolutions of $P_{\text {trans }}$ of $(5,3,3)$ and $(5,3,2)$ in Fig. 6 are very similar. The time evolutions of $P_{\text {trans }}$ of $(5,5,0)$ and $(5,5,1)$ in Fig. 6 and of $(10,10,0)$ and $(10,10,1)$ in Fig. 7 are almost indistinguishable.

The dynamics for $J=5$ shown in Fig. 6 displays similar trends: we do not see any strong effect of overall rotation. The shapes of $P_{\text {trans }}$ with $k_{a}^{\prime}$ and $k_{c}^{\prime}$ vary a little bit but the final values of the trans isomer are not very different from what is observed with $J=0$. The situation is similar when $J$ increases as shown in Fig. 7 and 8. The increase of $P_{\text {trans }}$ becomes rather substantial only for $J$ and $k_{a}^{\prime}$ large: see for instance $(10,10,0)$ and $(15,15,1)$ in Fig. 7 and 8 . But these eigenstates are not strongly populated, even at room temperature.

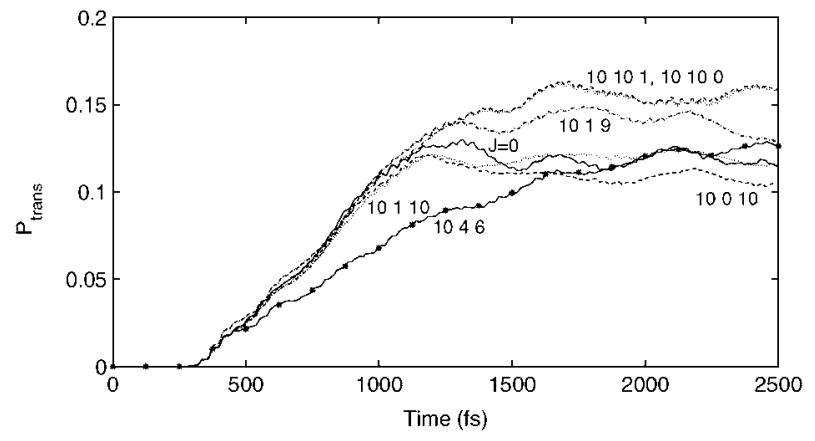

Fig. 7 Similar to Fig. 5 but for $J=10$ with the labels of Table 4 .

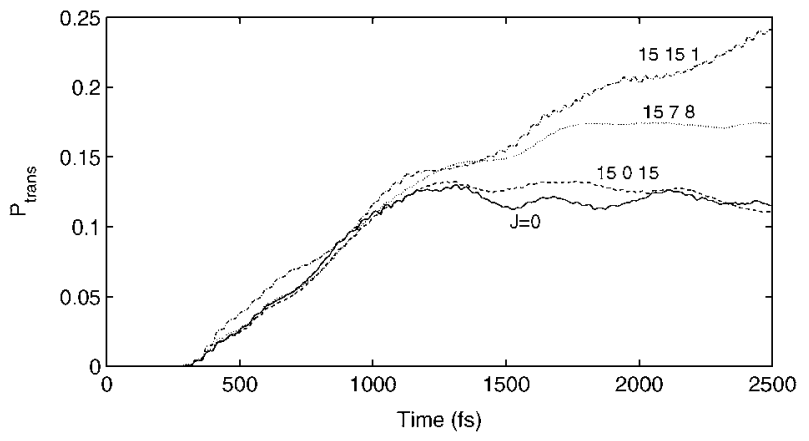

Fig. 8 Similar to Fig. 5 but for $J=15$ with the labels of Table 5 .

To conclude, the isomerization rates do not differ much. The effect of the Coriolis coupling is small and does not increase the isomerization rate appreciably for the process we are investigating. This result is interesting since it had been suggested by experimentalists that the isomerization of $\mathrm{HONO}$ could have been enhanced by overall rotation. Our simulations show that it is not the case. It is also an important result since it proves that the process described in ref. 20 is robust. In ref. 20, we had already checked the robustness of the mechanism by changing the values of the frequency of the pulse. We had also performed a simulation with a chirped pulse where the carrier frequency varies from $889 \mathrm{~cm}^{-1}$ to $850 \mathrm{~cm}^{-1}$. We have noticed that the same mechanism was induced and that similar isomerization ratios were obtained. The present work shows that the excitation process is robust against initial rotational excitations. We can predict that the process varies little with temperature for temperatures low enough that thermal excitation of the torsional mode is negligible.

In the present work, we have not taken into account the transitions from $J$ to $J^{\prime}$ with $J^{\prime} \neq J$ upon photon absorption. With the parameters used for our pulse, not many but some of them may occur, in particular those corresponding to a change of $J$ by 1 and a $\left|\Delta k_{a}\right|=1$ selection rule. It will result in different superpositions of the same eigenstates in the energy domain where the isomerization occurs (between 4000 and $6000 \mathrm{~cm}^{-1}$ ) but since they all do not increase the isomerization sensibly as shown by our simulations, it is highly probable that we will not see more isomerization if we include these transitions explicitly. However, it could be interesting to add these transitions in the future in our methodology, not to increase the isomerization of HONO, but to study the alignment of molecules by laser pluses in a systematic way.

\section{Conclusions and outlook}

We have implemented an exact form of the kinetic energy operator including overall rotation for a tetra-atomic molecule in non-orthogonal coordinates in the Heidelberg MCTDH package. This operator could be useful for many systems in the future. We have compared the experimental rovibrational eigenvalues available in the literature with those obtained with our PES: the agreement is excellent as for the comparison with the experimental vibrational eigenvalues published in ref. 20.

The effects of the Coriolis coupling are weak on the process studied in ref. 20. This process leads to the cis $\rightarrow$ trans 
isomerization of HONO. The excitation process is robust against variations of the excitation energy and we predict that the isomerization rate varies little with temperature.

In the present work, HONO was considered in the gas phase. Since the intrinsic rotational averaging that occurs when molecules freely rotate decreases the efficiency of the process, one efficient way to improve selectivity could be molecular orientation by laser alignment. Molecular orientation is a very active field of current research. ${ }^{54-59}$ In this context, it should be noticed that, in the present work, we have not taken into account the transitions from $J$ to $J^{\prime}$ with $J^{\prime} \neq J$ upon photon absorption. For this, it will be necessary to start from a laser pulse parallel to a Laboratory-Fixed frame and to implement the Euler matrices in the scalar product $-\hat{\vec{\mu}} \cdot \vec{E}(t)$. It will be then possible to mimic the experimental molecular orientation by laser "adiabatic" alignment with a longer laser pulse and to trigger the isomerization with a second laser pulse identical to the one used in the present work as in the experiments of Stapelfeldt and coworkers. ${ }^{60-62}$

\section{Appendix A: kinetic energy operator}

Here, we report the kinetic energy operator (KEO). As in ref. 19, we use the polyspherical approach to derive the kinetic energy operator. The polyspherical approach is a general method for deriving kinetic energy operators. ${ }^{39-42}$ This formalism, which is based on a polyspherical parametrization of a $\mathrm{N}$-atom system, can be applied whatever the number of atoms and whatever the set of vectors: Jacobi, Radau, valence, etc. As aforementioned, we choose here for HONO three valence vectors $\vec{R}_{1}=\overrightarrow{\mathrm{OH}}, \quad \vec{R}_{2}=\overrightarrow{\mathrm{ON}}$ (double bond), and $\vec{R}_{3}=\overrightarrow{\mathrm{ON}}$ (single bond) as depicted in Fig. 1. The three vectors are in turn parametrized by six polyspherical coordinates as shown in Fig. 1 of ref. 20. We use the same conventions as defined in Appendix A of ref. 19: i.e. $S_{1}=1, S_{2}=-1, \theta_{2}^{\text {new }}=\pi-\theta_{2}$, $\xi_{1}=\cos \left(\theta_{1}\right)$ and $\xi_{2}=\cos \left(\theta_{2}^{\text {new }}\right)$. In the present work, we include for the first time the overall rotation and the Coriolis coupling. Eqn (102), (C.4), (C.5), and (C.6) of ref. 42 lead to the following vibrational kinetic energy operator:

$$
\hat{T}=\hat{T}_{v i b}+\hat{T}_{r o t}+\hat{T}_{c o r} ;
$$

with

$$
\begin{aligned}
\hat{T}_{\mathrm{vib}}= & -\sum_{i=1}^{3} \frac{M_{i i}}{2} \frac{\partial^{2}}{\partial R_{i}^{2}}-\sum_{i=1}^{2}\left[S_{i} M_{i 3} \xi_{i}\left(\frac{\partial^{2}}{\partial R_{3} \partial R_{i}}+\frac{1}{R_{3} R_{i}}\right)\right. \\
& \left.+\left(\frac{M_{i i}}{2 R_{i}^{2}}+\frac{M_{33}}{2 R_{3}^{2}}\right) \frac{\partial}{\partial \xi_{i}}\left(1-\xi_{i}^{2}\right) \frac{\partial}{\partial \xi_{i}}\right] \\
& -\sum_{i=1}^{2}\left(\frac{S_{i} M_{i 3}}{2 R_{3}} \frac{\partial}{\partial R_{i}}+\frac{M_{i 3}}{2 R_{i}} \frac{\partial}{\partial R_{3}}\right) \\
& \times\left(\left(1-\xi_{i}^{2}\right) \frac{\partial}{\partial \xi_{i}}+\frac{\partial}{\partial \xi_{i}}\left(1-\xi_{i}^{2}\right)\right) \\
& +\sum_{i \neq j=1}^{2} \frac{S_{i} M_{i 3} \sqrt{1-\xi_{i}^{2}} \cos \tau}{2 R_{3}} \frac{\partial}{\partial R_{i}} \\
& \times\left(\sqrt{1-\xi_{j}^{2}} \frac{\partial}{\partial \xi_{j}}+\frac{\partial}{\partial \xi_{j}} \sqrt{1-\xi_{j}^{2}}\right)
\end{aligned}
$$

$$
\begin{aligned}
& +\frac{M_{33} \cos \tau}{2 R_{3}^{2}}\left(\sqrt{1-\xi_{1}^{2}} \frac{\partial^{2}}{\partial \xi_{1} \partial \xi_{2}} \sqrt{1-\xi_{2}^{2}}\right. \\
& \left.+\sqrt{1-\xi_{2}^{2}} \frac{\partial^{2}}{\partial \xi_{1} \partial \xi_{2}} \sqrt{1-\xi_{1}^{2}}\right) \\
& +\sum_{i \neq j=1}^{2} \frac{S_{i} M_{i 3}}{R_{3} R_{i}} \frac{\partial}{\partial \xi_{i}}\left(1-\xi_{i}^{2}\right) \xi_{i} \frac{\partial}{\partial \xi_{i}} \\
& +\frac{S_{i} M_{i 3} \xi_{j} \sqrt{1-\xi_{i}^{2}}}{2 R_{3} \sqrt{1-\xi_{j}^{2}}} \frac{\partial}{\partial R_{i}}\left(\sin \tau \frac{\partial}{\partial \tau}+\frac{\partial}{\partial \tau} \sin \tau\right) \\
& -\sum_{i \neq j=1}^{2} \frac{S_{i} M_{i 3} \cos \tau}{2 R_{3} R_{i}}\left(\sqrt{1-\xi_{j}^{2}} \frac{\partial^{2}}{\partial \xi_{j} \partial \xi_{i}} \sqrt{1-\xi_{i}^{2}}\right. \\
& \left.+\sqrt{1-\xi_{i}^{2}} \frac{\partial^{2}}{\partial \xi_{j} \partial \xi_{i}} \sqrt{1-\xi_{j}^{2}}\right) \\
& -\sum_{i \neq j=1}^{2} \frac{S_{i} M_{i 3} \xi_{j}}{2 R_{3} R_{i} \sqrt{1-\xi_{j}^{2}}} \\
& \times\left(\sin \tau \frac{\partial^{2}}{\partial \xi_{i} \partial \tau} \xi_{i} \sqrt{1-\xi_{i}^{2}}+\xi_{i} \sqrt{1-\xi_{i}^{2}} \frac{\partial^{2}}{\partial \xi_{i} \partial \tau} \sin \tau\right) \\
& +\sum_{i \neq j=1}^{2}\left(\frac{M_{33} \xi_{i}}{2 R_{3}^{2} \sqrt{1-\xi_{i}^{2}}}-\frac{S_{i} M_{i 3}}{2 R_{3} R_{i} \sqrt{1-\xi_{i}^{2}}}\right) \\
& \times\left(\sin \tau \frac{\partial^{2}}{\partial \xi_{i} \partial \tau} \sqrt{1-\xi_{j}^{2}}+\sqrt{1-\xi_{i}^{2}} \frac{\partial^{2}}{\partial \xi_{i} \partial \tau} \sin \tau\right) \\
& \left.-\sum_{i=1}^{2} \frac{M_{i i}}{2 R_{i}^{2}\left(1-\xi_{i}^{2}\right)}+\frac{M_{33} \xi_{i}^{2}}{2 R_{3}^{2}\left(1-\xi_{i}^{2}\right)}\right)
\end{aligned}
$$$$
-\frac{S_{i} M_{i 3} \xi_{i}}{R_{3} R_{i}\left(1-\xi_{i}^{2}\right)} \frac{\partial^{2}}{\partial \tau^{2}}+\left(\sum_{i \neq j=1}^{2} \frac{S_{i} M_{i 3} \xi_{j}}{R_{3} R_{i} \sqrt{1-\xi_{j}^{2}} \sqrt{1-\xi_{i}^{2}}}\right.
$$$$
\left.-\frac{M_{33} \xi_{2} \xi_{1}}{R_{3}^{2} \sqrt{1-\xi_{2}^{2}} \sqrt{1-\xi_{1}^{2}}}\right) \frac{\partial}{\partial \tau} \cos \tau \frac{\partial}{\partial \tau}
$$$$
\hat{T}_{\text {rot }}=\frac{1}{1-\xi_{2}^{2}}\left[\frac{M_{22}}{2 R_{2}^{2}}+\frac{M_{23} \xi_{2}}{R_{2} R_{3}}+\frac{M_{33} \xi_{2}^{2}}{2 R_{3}^{2}}\right] J_{z^{\mathrm{BF}}}^{2}+\frac{M_{33}}{2 R_{3}^{2}}\left(J^{2}-J_{z^{\mathrm{BF}}}\right)
$$$$
-\frac{1}{2 \sqrt{1-\xi_{2}^{2}}}\left(\frac{M_{23}}{R_{2} R_{3}}+\frac{M_{33} \xi_{2}}{R_{3}^{2}}\right)\left(J_{x^{\mathrm{BF}}} J_{z^{\mathrm{BF}}}+J_{z^{\mathrm{BF}}} J_{x^{\mathrm{BF}}}\right),
$$ 
and

$$
\begin{aligned}
& \hat{T}_{\text {cor }}=-\frac{i M_{23} \sqrt{1-\xi_{2}^{2}}}{R_{3}} J_{y} \frac{\partial}{\partial R_{2}}+\frac{i M_{13} \sqrt{1-\xi_{1}^{2}}}{R_{3}} \\
& \left(\sin \tau J_{x^{\mathrm{BF}}}-\cos \tau J_{y^{\mathrm{BF}}}-\frac{\xi_{2} \sin \tau}{\sqrt{1-\xi_{2}^{2}}} J_{z^{\mathrm{BF}}}\right) \frac{\partial}{\partial R_{1}} \\
& -\frac{i M_{13}}{2 R_{1} R_{3}}\left(\sin \tau J_{x^{\mathrm{BF}}}-\cos \tau J_{y^{\mathrm{BF}}}-\frac{\xi_{2} \sin \tau}{\sqrt{1-\xi_{2}^{2}}} J_{z^{\mathrm{BF}}}\right) \\
& \times\left(\xi_{1} \sqrt{1-\xi_{1}^{2}} \frac{\partial}{\partial \xi_{1}}+\frac{\partial}{\partial \xi_{1}} \xi_{1} \sqrt{1-\xi_{1}^{2}}\right) \\
& +\frac{i M_{33}}{2 R_{3}^{2}}\left(\sin \tau J_{x^{\mathrm{BF}}}-\cos \tau J_{y^{\mathrm{BF}}}-\frac{\xi_{2} \sin \tau}{\sqrt{1-\xi_{2}^{2}}} J_{z^{\mathrm{BF}}}\right) \\
& \times\left(\sqrt{1-\xi_{1}^{2}} \frac{\partial}{\partial \xi_{1}}+\frac{\partial}{\partial \xi_{1}} \sqrt{1-\xi_{1}^{2}}\right) \\
& -\frac{i M_{23} \sin \tau}{2 R_{2} R_{3} \sqrt{1-\xi_{2}^{2}}} J_{z^{\mathrm{BF}}}\left(\sqrt{1-\xi_{1}^{2}} \frac{\partial}{\partial \xi_{1}}+\frac{\partial}{\partial \xi_{1}} \sqrt{1-\xi_{1}^{2}}\right) \\
& +\frac{i M_{23}}{2 R_{2} R_{3}} J_{y^{\mathrm{BF}}}\left(\xi_{2} \sqrt{1-\xi_{2}^{2}} \frac{\partial}{\partial \xi_{2}}+\frac{\partial}{\partial \xi_{2}} \xi_{2} \sqrt{1-\xi_{2}^{2}}\right) \\
& +\frac{i M_{33}}{2 R_{3}^{2}} J_{y^{\mathrm{BF}}}\left(\sqrt{1-\xi_{2}^{2}} \frac{\partial}{\partial \xi_{2}}+\frac{\partial}{\partial \xi_{2}} \sqrt{1-\xi_{2}^{2}}\right) \\
& -\frac{i}{\sqrt{1-\xi_{1}^{2}}}\left[\left(-\frac{M_{13}}{2 R_{1} R_{3}}+\frac{M_{33} \xi_{1}}{2 R_{3}^{2}}\right)\left(J_{x}-\frac{\xi_{2} J_{z^{\mathrm{BF}}}}{\sqrt{1-\xi_{2}^{2}}}\right)\right. \\
& \left.-\frac{M_{23} \xi_{1}}{2 R_{2} R_{3} \sqrt{1-\xi_{2}^{2}}} J_{z^{\mathrm{BF}}}\right] \\
& \times\left(\cos \tau \frac{\partial}{\partial \tau}+\frac{\partial}{\partial \tau} \cos \tau\right) \\
& -\frac{i}{\sqrt{1-\xi_{2}^{2}}}\left[-\left(\frac{2 M_{23} \xi_{2}}{R_{2} R_{3}}+\frac{M_{22}}{R_{2}^{2}}+\frac{M_{33} \xi_{2}^{2}}{R_{3}^{2}}\right) \frac{J_{z^{\mathrm{BF}}}}{\sqrt{1-\xi_{2}^{2}}}\right. \\
& \left.+\left(\frac{M_{23}}{R_{2} R_{3}}+\frac{M_{33} \xi_{2}}{R_{3}^{2}}\right) J_{x^{\mathrm{BF}}}\right] \frac{\partial}{\partial \tau} \\
& -\left(-\frac{M_{13}}{2 R_{1} R_{3}}+\frac{M_{33} \xi_{1}}{2 R_{3}^{2}}\right) \frac{i J_{y^{\mathrm{BF}}}}{\sqrt{1-\xi_{1}^{2}}}\left(\sin \tau \frac{\partial}{\partial \tau}+\frac{\partial}{\partial \tau} \sin \tau\right) \text {. }
\end{aligned}
$$

The matrix elements $M_{i j}$ are given as

$$
\mathbf{M}=\left(\begin{array}{ccc}
\frac{1}{m_{H}}+\frac{1}{m_{O}} & 0 & \frac{1}{m_{O}} \\
0 & \frac{1}{m_{O}}+\frac{1}{m_{N}} & -\frac{1}{m_{N}} \\
\frac{1}{m_{O}} & -\frac{1}{m_{N}} & \frac{1}{m_{O}}+\frac{1}{m_{N}}
\end{array}\right) .
$$

The Body-Fixed (BF) frame is defined such that the $z^{\mathrm{BF}}$ axis is parallel to $\vec{R}_{3}=\overrightarrow{\mathrm{ON}}$ (see Fig. 1) and such that $\vec{R}_{2}$ is parallel to the $\left(\left(x^{\mathrm{BF}} G z^{\mathrm{BF}}\right), x^{\mathrm{BF}}>0\right)$ half-plane. It should be emphasized that the BF-axes are different from the principal axes of inertia.

$J_{z}$ acts on the third Euler angles and becomes formally in the kinetic energy operator (KEO) the multiplicative operator $k$, the projection of $\vec{J}$ onto the $z^{\mathrm{BF}}$ axis. $J_{x^{B F}}$ and $J_{y^{B F}}$ operate on the variable $k$, with

$$
\begin{aligned}
& J_{x^{\mathrm{BF}}}=\left(J_{+}+J_{-}\right) / 2, \\
& J_{y^{\mathrm{BF}}}=\left(J_{+}-J_{-}\right) /(2 i)
\end{aligned}
$$

and $J_{ \pm}$gives $k \rightarrow k \pm 1$.

\section{Acknowledgements}

The authors thank Prof. M. Herman for providing the experimental rovibrational eigenenergies. Financial support through the German and French science foundations through contact DFG/ANR-09-BLAN-0417 is also gratefully acknowledged.

\section{References}

1 Femtochemistry, ed. M. Chergui, World Scientific, Singapore, 1996.

2 A. H. Zewail, Femtochemistry - Ultrafast Dynamics of the Chemical Bond, World Scientific, Singapore, 1994.

3 H. Ihee, V. Lobastov, U. Gomez, B. Goodson, R. Srinivasan, C.-Y. Ruan and A. H. Zewail, Science, 2001, 291, 385.

4 A. H. Zewail, Femtochemistry, ed. F. C. De Schryver, S. De Feyter and G. Schweitzer, Wiley-VCH, 2001.

5 M. Quack, Аnпи. Rev. Phys. Chem., 1990, 41, 839.

6 K. K. Lehmann, G. Scoles and B. H. Pate, Annu. Rev. Phys. Chem., 1994, 45, 241.

7 D. Nesbitt and R. Field, J. Phys. Chem., 1996, 100, 12735

8 G. A. Worth, M. H. Beck, A. Jäckle and H.-D. Meyer, The MCTDH Package, Version 8.2, 2000; H.-D. Meyer, Version 8.3 (2002), Version 8.4 (2007). See http://mctdh.uni-hd.de/.

9 H.-D. Meyer, U. Manthe and L. S. Cederbaum, The multi-configurational time-dependent Hartree approach, Chem. Phys. Lett., 1990, 165, 73-78.

10 M. H. Beck, A. Jäckle, G. A. Worth and H.-D. Meyer, The multiconfiguration time-dependent Hartree (MCTDH) method: A highly efficient algorithm for propagating wave packets, Phys. Rep., 2000, 324, 1-105.

11 H.-D. Meyer and G. A. Worth, Quantum molecular dynamics: Propagating wavepackets and density operators using the multiconfiguration time-dependent Hartree (MCTDH) method, Theor. Chem. Acc., 2003, 109, 251-267.

12 Multidimensional Quantum Dynamics: MCTDH Theory and Applications, ed. H.-D. Meyer, F. Gatti and G. A. Worth, Wiley-VCH, Weinheim, 2009.

13 F. Gatti and H.-D. Meyer, Intramolecular vibrational energy redistribution in Toluene: A nine dimensional quantum mechanical study using the MCTDH algorithm, Chem. Phys., 2004, 304, 3-15.

14 C. Iung, F. Gatti and H.-D. Meyer, Intramolecular vibrational energy redistribution in the highly excited fluoroform molecule: A quantum mechanical study using the MCTDH algorithm, J. Chem. Phys., 2004, 120, 6992-6998.

15 G. Pasin, F. Gatti, C. Iung and H.-D. Meyer, Theoretical investigation of Intramolecular Vibrational Energy Redistribution in highly excited HFCO, J. Chem. Phys., 2006, 124, 194304.

16 G. Pasin, C. Iung, F. Gatti and H.-D. Meyer, Theoretical investigation of highly excited vibrational states in DFCO: Calculation of the out-of-plane bending states and simulation of the intramolecular vibrational energy redistribution, J. Chem. Phys., 2007, 126, 024302.

17 H.-D. Meyer, F. Le Quéré, C. Léonard and F. Gatti. Calculation, Selective population of vibrational levels with the Multiconfiguration Time-Dependent Hartree (MCTDH) algorithm, Chem. Phys., 2006, 329, 179-192. 
18 F. Richter, M. Hochlaf, P. Rosmus, F. Gatti and H.-D. Meyer, A study of mode-selective trans-cis isomerisation in HONO using ab initio methodology, J. Chem. Phys., 2004, 120, 1306-1317.

19 F. Richter, P. Rosmus, F. Gatti and H.-D. Meyer, Time-dependent wavepacket study on trans-cis isomerisation of HONO, J. Chem. Phys., 2004, 120, 6072-6084.

20 F. Richter, F. Gatti, C. Léonard, F. Le Quéré and H.-D. Meyer, Time-dependent wave packet study on trans-cis isomerisation of HONO driven by an external field, J. Chem. Phys., 2007, 127, 164315.

21 P. A. McDonald and J. S. Shirk, The infrared laser photoisomerization of HONO in solid $\mathrm{N}_{2}$ and Ar, J. Chem. Phys., 1982, 77, 2355.

22 A. E. Shirk and J. S. Shirk, Isomerization of HONO in solid nitrogen by selective vibrational excitation, Chem. Phys. Lett., 1983, 97, 549-552.

23 L. Khriatchev, J. Lundell, E. Isoniemi and M. Räsänen, HONO in solid $\mathrm{Kr}$ : Site-selective trans-cis isomerization with narrow-band infrared radiation, J. Chem. Phys., 2000, 113, 4265-4273.

24 J. M. Guilmot, M. Carleer, M. Godefroid and M. Herman, J. Mol. Spectrosc., 1990, 143, 81.

25 F. Mélen and M. Hermann, J. Phys. Chem. Ref. Data, 1992, 21, 831 .

26 J. M. Guilmot, M. Godefroid and M. Herman, The rovibrational spectrum of trans- $\mathrm{HNO}_{2}$, J. Mol. Spectrosc., 1993, 160, 387-400.

27 J. M. Guilmot, F. Mélen and M. Herman, The rovibrational spectrum of cis- $\mathrm{HNO}_{2}$, J. Mol. Spectrosc., 1993, 160, 401-410.

28 F. Reiche, B. Abel, R. D. Beck and T. R. Rizzo, J. Chem. Phys., $2000, \mathbf{1 1 2}, 8885$.

29 Y. Guo and D. L. Thompson, A theoretical study of cistrans isomerization in hono using an empirical valence bond potential, J. Chem. Phys., 2003, 118, 1673-1678.

30 Y. Guo and D. L. Thompson, J. Chem. Phys., 2004, 120, 898.

31 A. Dehayem-Kamadjeu, O. Pirali, J. Orphal, I. Kleiner and P.-M. Flaud, J. Mol. Spectrosc., 2005, 234, 182-189.

32 R. Schanz, V. Botan and P. Hamm, A femtosecond study of the infrared-driven cis-trans isomerization of nitrous acid (HONO), J. Chem. Phys., 2005, 122, 044509.

33 V. Botan, R. Schanz and P. Hamm, The infrared-driven cis-trans isomerization of HONO. II: Vibrational relaxation and slow isomerization channel, J. Chem. Phys., 2006, 124, 234511.

$34 \mathrm{~V}$. Botan and P. Hamm, Intramolecular vibrational energy relaxation in nitrous acid (HONO), J. Chem. Phys., 2008, 129, 234511.

$35 \mathrm{~V}$. Botan and P. Hamm, Temperature dependence of the IR driven cis-trans isomerization of nitrous acid (HONO), J. Chem. Phys., 2008, 129, 114510 .

36 V. Botan and P. Hamm, Rotational dynamics of nitrous acid (HONO) in Kr matrix, J. Chem. Phys., 2008, 129, 044507.

$37 \mathrm{P}$. Hamm, The infrared-driven cis-trans isomerization of nitrous acid HONO. III: A mixed quantum-classical simulation, Chem. Phys., 2008, 347, 503.

38 H. M. Le and L. M. Raff, Cis to trans, trans to cis isomerizations and $\mathrm{N}-\mathrm{O}$ bond dissociation of nitrous acid (HONO) on an $a b$ initio potential surface obtained by novelty sampling and feed-forward neural network fitting, J. Chem. Phys., 2008, 128, 194310.

39 F. Gatti, C. Iung, M. Menou, Y. Justum, A. Nauts and $\mathrm{X}$. Chapuisat, Vector parametrization of the $n$-atom problem in quantum mechanics. I. Jacobi vectors, J. Chem. Phys., 1998, 108, 8804 .

40 F. Gatti and C. Iung, Exact and constrained kinetic energy operators in polyspherical coordinates, J. Theor. Comput. Chem., 2003, 2, 507-522.
41 C. Iung and F. Gatti, Polyspherical parametrization of a $n$-atom system: Principles and applications, Int. J. Quantum Chem., 2006, 106, 130.

42 F. Gatti and C. Iung, Exact and constrained kinetic energy operators for polyatomic molecules: The polyspherical approach, Phys. Rep., 2009, 484, 1-69.

43 D. Lauvergnat and A. Nauts, Exact numerical computation of a kinetic energy operator in curvilinear coordinates, J. Chem. Phys., 2002, 116, 8560.

44 R. Loudon, The Quantum Theory of Light, Oxford University Press, Oxford, 1983.

45 F. A. Hamprecht, A. J. Cohen, D. J. Tozer and N. C. Handy, J. Chem. Phys., 1998, 109, 6264.

46 R. D. Amos, I. L. Alberts, J. S. Andrews, S. M. Colwell, N. C. Handy, D. Jayatilaka, P. J. Knowles, R. Kobayashi, K. E. Laidig, G. Laming, A. M. Lee, P. E. Maslen, C. W. Murray, J. E. Rice, E. D. Simandiras, A. J. Stone, M.-D. Su and D. J. Tozer, The Cambridge Analytic Derivatives Package, Issue 6.5, 2001. See http://www-theor.ch.cam.ac.uk/soft ware/cadpac.html.

47 C. Eckart, Some studies concerning rotating axes and polyatomic molecules, Phys. Rev., 1935, 47, 552-558.

48 L. J. Doriol, F. Gatti, C. Iung and H.-D. Meyer, Computation of vibrational energy levels and eigenstates of fluoroform using the multiconfiguration time-dependent Hartree method, J. Chem. Phys., 2008, 129, 224109.

49 E. Wilson, J. Decius and P. Cross, Molecular Vibrations, McGraw-Hill, New York, 1955.

50 P. R. Bunker and P. Jensen, Molecular Symmetry and Spectroscopy, NRC Research Press, 2nd edn, 1998.

51 R. N. Zare, Angular momentum, Wiley, New York, 1988.

52 F. Gatti, Vector parametrization of the $n$-atom problem in quantum mechanics. III. Separation into two sub-systems, J. Chem. Phys., 1999, 111, 7225.

53 B. Lasorne, M. A. Robb, H.-D. Meyer and F. Gatti, The electronic excited states of ethylene with large-amplitude deformations: A dynamical symmetry group investigation, Chem. Phys., 2010, 377, 30-45.

54 H. J. Loesch and A. Reimscheid, J. Chem. Phys., 1990, 93, 4779.

55 A. Slenszka, B. Friedrich and D. Herschbach, Phys. Rev. Lett., 1994, 72, 1806.

56 W. Kim and P. M. Felker, J. Chem. Phys., 1996, 104, 1147.

57 L. Cai, J. Marango and B. Friedrich, Phys. Rev. Lett., 2001, 86, 775.

58 T. Seideman, On the dynamics of rotationally-broad, spatiallyaligned wavepackets, J. Chem. Phys., 2001, 115, 5965.

59 R. Marquardt, M. Quack, I. Thanopoulos and D. Luckhaus, Tunneling dynamics of the NH chromophore in $\mathrm{NHD}_{2}$ during and after coherent infrared excitation, J. Chem. Phys., 2003, 118, 643-658

60 C. B. Madsen, L. B. Madsen, S. S. Viftrup, M. P. Johansson, T. B. Poulsen, L. Holmegaard, V. Kumarappan, K. A. Jorgensen and $\mathrm{H}$. Stapelfeldt, A combined experimental and theoretical study on realizing and using laser controlled torsion of molecules, J. Chem. Phys., 2009, 130, 234310.

61 C. B. Madsen, L. B. Madsen, S. S. Viftrup, M. P. Johansson, T. B. Poulsen, L. Holmegaard, V. Kumarappan, K. A. Jorgensen and H. Stapelfeldt, Manipulating the torsion of molecules by strong laser pulses, Phys. Rev. Lett., 2009, 102, 073007.

62 J. L. Hansen, H. Stapelfeldt, D. Dimitrovski, M. Abu-sanha, C. P. J. Chrisitan and L. B. Madsen, Time-resolved photoelectron angular distributions from strong-field ionization of rotating naphthalene molecules, Phys. Rev. Lett., 2011, 106, 073001. 